\title{
Low Perioperative Serum Prealbumin Predicts Early Recurrence After Curative Pulmonary Resection for Non-Small-Cell Lung Cancer
}

\author{
Francesco Cavallin • Marco Scarpa • \\ Matteo Cagol · Rita Alfieri · Carlo Castoro
}

Published online: 6 February 2013

(c) Société Internationale de Chirurgie 2013

To the Editor

We read with great interest the article by Kawai and Ota [1], recently published in World Journal of Surgery. They evaluated the relationship between the perioperative nutritional status and postoperative early recurrence of lung cancer, claiming a significant risk effect of low perioperative serum prealbumin.

The serum level of prealbumin was used as an indicator of nutritional status, and the authors split their study sample into two groups: patients with preoperative prealbumin $<23 \mathrm{mg} / \mathrm{dl}$ and postoperative prealbumin $<15 \mathrm{mg} / \mathrm{dl}$ were included in the "low-prealbumin" group.

The dichotomization of continuous variables is a common approach in clinical research because of its perceived advantages. It exemplifies the presentation and the interpretation of the results, because the two groups represent "low" and "high" levels of serum prealbumin, and so the features observed in the "low" group are indirectly linked by the reader to the effect of a decrease of serum prealbumin (and vice versa). This approach also exemplifies the statistical analysis, leading to comparisons between the two groups; in the simplest case, such comparisons are usually performed by Student's $t$-test and the $\chi^{2}$ test, which are familiar to most researchers.

However, dichotomizing continuous variables leads to several problems that often are underestimated [2,3]. For example, the variation in outcome between groups may be underestimated because the variability may be subsumed within each group. The possible non-linearity relationship

F. Cavallin $(\bowtie) \cdot$ M. Scarpa · M. Cagol · R. Alfieri ·

C. Castoro

Oncological Surgery Unit, Veneto Institute of Oncology

(IOV-IRCCS), Padova, Italy

e-mail: cescocava@libero.it between serum prealbumin and outcome could be hidden by the above-mentioned binary split. It also defines an unrealistic scenario, because subjects belonging to the same group are considered similar even if their difference in serum prealbumin could suggest otherwise. In the same way, subjects close to but on the opposite side of the cutpoint are described as having a different outcome rather than a similar one. It causes loss of information and a consequent decrease in statistical power.

In their study, Kawai and Ota defined cutoff values of serum prealbumin to achieve more practical criteria for defining impaired nutritional status. We agree that a cutoff may be easy to use by clinicians, but these authors' choice was based on their results, and it was completely arbitrary. Nevertheless, there is no reason to suppose that a underlying dichotomy in serum albumin exists; it would be like a threshold that changes the risk status of patients crossing it.

In their study, Kawai and Ota provide useful information about the role of serum prealbumin as a prognostic marker, but we think that serum prealbumin —and age too-should have been included in the multivariable analysis as continuous variables and the identification of possible cutoffs should be left to further studies with appropriate mathematical methods.

\section{References}

1. Kawai H, Ota H (2012) Low perioperative serum prealbumin predicts early recurrence after curative pulmonary resection for non-small-cell lung cancer. World J Surg 36:2853-2857

2. Royston P, Altman DG, Sauerbrei W (2006) Dichotomizing continuous predictors in multiple regression: a bad idea. Stat Med 25:127-141

3. Altman DG, Royston P (2006) The cost of dichotomising continuous variables. BMJ 332:1080 